\title{
Adaptive Bolus-Based Set-Point Regulation of Hyperglycemia in Critical Care
}

\author{
J. G. Chase ${ }^{1}$, G. M. Shaw ${ }^{2}$, J. Lin ${ }^{1}$, C. V. Doran ${ }^{1}$, C. E. Hann ${ }^{1}$, M. B. Robertson ${ }^{1}$, P. M. Browne ${ }^{1}$, T. \\ Lotz $^{1}$, G. C. Wake ${ }^{3}$, B. Broughton ${ }^{3}$ \\ ${ }^{1}$ Department of Mechanical Engineering, University of Canterbury, New Zealand \\ ${ }^{2}$ Department of Intensive Care Medicine, Christchurch Hospital, New Zealand \\ ${ }^{3}$ Department of Mathematics and Statistics, University of Canterbury, New Zealand
}

\begin{abstract}
Critically ill patients are often hyperglycemic and extremely diverse in their dynamics. Consequently, fixed protocols and sliding scales can result in error and poor control. A two-compartment glucose-insulin system model that accounts for time-varying insulin sensitivity and endogenous glucose removal, along with two different saturation kinetics is developed and verified in proof-of-concept clinical trials for adaptive control of hyperglycemia. The adaptive control algorithm monitors the physiological status of a critically ill patient, allowing real-time tight glycemic regulation. The bolus-based insulin administration approach is shown to result in safe, targeted stepwise glycemic reduction for three critically ill patients.
\end{abstract}

Keywords-Adaptive control, critical care, glucose-insulin system model, hyperglycemia, insulin bolus

\section{INTRODUCTION}

Critically ill patients often experience stress-induced hyperglycemia and severe insulin resistance without history of diabetes (e.g. [1 7]). Hyperglycemia is not only a marker for severity of illness, it also worsens outcomes (e.g. [1, 5]). Van den Berghe et al. [5, 6] have shown that tight glucose control can reduce Intensive Care Unit (ICU) patient mortality by $45 \%$ if the glucose level is kept less than 6.1 $\mathrm{mmol} / \mathrm{L}$ for a cardiac care population. Krinsley [7] showed a $6 \%$ total reduction in mortality over a broader ICU population with a higher glucose limit of $7.75 \mathrm{mmol} / \mathrm{L}$. In addition, because automated glucose sensors are still in their infancy, initial implementations of automated glycemic control will likely be in a clinical environment. Automated control algorithms capable of tight regulation for glucose intolerant ICU patients would reduce mortality, as well as the current burden on ICU medical resources and time.

The proposed control algorithm needs to reduce elevated blood glucose levels in a controlled, predictable manner, while accounting for inter-patient variability and varying physiological condition. Hence, it must be adaptive and/or able to identify changes in patient dynamics, particularly with respect to insulin sensitivity. Previous works, such as Hovorka et al. [8], are primarily concerned with Type 1 diabetes and treatment of ambulatory individuals. The primary difference between this study and previous works that involve controlled insulin infusion experiments [8 10] is the focus on critical care patients, which can introduce significant additional dynamics due to illness, drug therapy and the impact of endogenous glucose removal.

\section{MAthematical Model}

Tight glucose control requires a patient-specific glucose-insulin system model that captures the fundamental dynamic responses to elevated glycemic levels and insulin. Doran et al. [10] used the minimal model by Bergman et al. [11], but failed to capture all transient dynamics, in particular the long term effect of exogenous insulin. Based on these results, an extended model was developed.

$$
\begin{gathered}
\dot{G}=p_{G} G-S_{I}\left(G+G_{E}\right) \frac{Q}{1+\alpha_{G} Q}+P(t) \\
Q=k \int_{0}^{t} I(\tau) e^{-k(t-\tau)} d \tau \\
\dot{I}=-n \frac{I}{1+\alpha_{I} I}+\frac{u_{e x}(t)}{V}
\end{gathered}
$$

where $G$ and $I$ denote the glucose above an equilibrium level under constant feed, $G_{E}$, and the plasma insulin from an exogenous insulin input respectively. Insulin utilization over time is represented by $Q$, with $k$ accounting for the effective insulin half-life in the system, which is set to $70 \mathrm{~min}$ as a median of a range of reported values [12, 13]. It can be adapted during a trial to better represent observed patient dynamics. Patient endogenous glucose removal and insulin sensitivity are $p_{G}$ and $S_{I}$ respectively. The parameter $V$ is the insulin distribution volume and $n$ is the first order decay rate for plasma insulin. External nutrition and insulin input are $P(t)$ and $u_{e x}(t)$ respectively. Michaelis-Menten parameters $\alpha_{I}$ and $\alpha_{G}$ respectively define the plasma insulin disappearance saturation and the insulin-stimulated glucose removal saturation.

Many studies have investigated insulin saturation in vivo (e.g. [12 14]). Two saturation mechanisms are included in (1) and (3), one for insulin action, the other for insulin transport from plasma to interstitial sites. The parameter, $\alpha_{I}$, in (3) bounds the plasma insulin disappearance rate. First order plasma insulin disappearance rate at low insulin concentration, $n$, is set to $0.16 \mathrm{~min}^{-1}$, a value that is fairly consistent across many studies (e.g. [14, 15]). The value of $\alpha_{I}$ is set to $0.0017 \mathrm{~L} / \mathrm{mU}$, which is also consistent across many studies (e.g. $[14,15])$.

Saturation in insulin-stimulated glucose removal has been evidenced in several clinical investigations (e.g. [15, 16]). The parameter, $\alpha_{G}$, in (1) defines the insulin-stimulated 
glucose removal saturation. This study uses $\alpha_{G}=1 / 65 \mathrm{~L} / \mathrm{mU}$ as an initial conservative choice from previous reported results (e.g. $[15,16])$, to ensure that saturation is more likely to be underestimated.

The model does not include the endogenous insulin production rate because it can be highly variable and difficult to obtain quickly in critical care, even though it is fairly consistent across the non-critically ill population (e.g. $[8,11,15])$. Therefore, the effect of endogenous insulin is combined with the effect of glucose to enhance glucose removal and inhibit endogenous glucose production for these short trials. The result is a time-varying parameter, $p_{G}$ that represents the body's ability to regulate blood glucose without exogenous insulin, or more simply, metabolic glucose resistance. The added saturation mechanism on insulin effect in (1) to (3) creates a unique index of insulin sensitivity, $S_{I}$ in contrast to other model-based measures (e.g. $[16,17])$. This approach allows $S_{I}$ to more closely approximate the true tissue sensitivity to insulin.

\section{PARAmeter Fitting AND Control Method}

Using generic values for $\alpha_{G}, \alpha_{l}, n, k$ and $V$ limits the only unknowns in the model to $p_{G}$ and $S_{I}$. This study utilizes an integration-based method developed by Hann and Hickman [18] to achieve high accuracy real-time tracking in $p_{G}$ and $S_{I}$. Both $p_{G}$ and $S_{I}$ are defined as first order piecewise linear to reduce computational intensity while capturing slow variations. The method results in a simple convex least squares problem that demands little computational time and intensity, in contrast to the commonly used non-linear recursive least squares routine that is non-convex and starting point dependent [19]. In addition, this approach is insensitive to measurement noise, a problem with gradientbased [10], as it effectively low-pass filters the data in the numerical integration summations. Constraints are placed on both parameters to ensure they are within the physiologically valid range (e.g. [8, 12, 13, 16, 17, 20 22]).

Prior to making an injection to achieve a corresponding blood glucose target, $p_{G}$ and $S_{I}$ are fitted using prior data. The interval between injections is one hour in this research. Prediction for the glucose profile one hour onwards is made by assuming the final values of $p_{G}$ and $S_{I}$ remain constant during following hour. The required bolus size to achieve the target blood glucose level is then determined using a bisection method and (1)-(3).

Insulin sensitivity can trade off with insulin effective half-life and its effective saturation limit. When the effective insulin half-life differs by greater than $50 \%$ from the originally assumed value of $70 \mathrm{~min}$, the impact is transferred into the value of $S_{I}$ and results in non-physiological saw tooth profiles.

Adapting the insulin half-life, or $k$, restores more constant and physiologically valid variations in $S_{I}$. The glucose removal saturation parameter, $\alpha_{G}$, is kept constant.
Adapting the saturation level in real-time is difficult because its detection is available only when significant saturation occurs, followed by large target error. For patient safety, $\alpha_{G}$ is set to $1 / 65 \mathrm{~L} / \mathrm{mU}$, corresponding to the highest reported saturation level found [13] to avoid the risk of administering excess insulin, due to seeing the patient as being at the saturation limit.

For effective control, ineffective delayed insulin, which is unused due to saturation, is always maintained below 30 $\mathrm{mU} / \mathrm{L}$ because of the extremely limited effect of any further insulin input. This limitation, in turn, limits the achievable glucose reduction. When the desired reduction reaches beyond this boundary, the target is reset to an achievable value, preventing unnecessary insulin being given to the patients and adding another level of safety.

\section{Clinical trial Methodology}

The proof-of-concept clinical trials consist of an insulin challenge hour followed by four hours of tight glycemic control. Each blood glucose target to be achieved one hour onwards is set at the end of that hour, targeting a 10 to $20 \%$ hourly reduction. The minimum target level is $4.5 \mathrm{mmol} / \mathrm{L}$. Ethical consent was obtained from the Canterbury Ethics Committee.

The trial begins at 0700 hours, at which time any insulin infusion is held constant with known constant naso-gastric feed maintained throughout the trial. Blood glucose is monitored hourly until 1000 hours to determine the patients' equilibrium blood glucose level, $G_{E}$. At 1000 hours, patients are injected with a fixed $1500 \mathrm{mU}$ Actrapid $^{\mathrm{TM}}$ insulin bolus via an intravenous cannula using a Graseby 3500 syringe pump. Plasma glucose is measured at 15-minute intervals until 1100 hours. Paired blood samples are taken and analyzed using a bedside Glucocard ${ }^{\mathrm{TM}}$ Test Strip II glucose testing kit, which has 7\% error [23].

Patient specific parameters, $p_{G}$ and $S_{I}$, are fitted using the first hour of data. Based on these values, an insulin bolus size is calculated by the controller to achieve the targeted glycemic reduction. Blood glucose is monitored every 30 minutes, and patient specific parameters are refit every hour using the data obtained in the previous hour. Following each refit, the controller determines the insulin bolus required to achieve the targeted glycemic reduction. Hence, the overall approach is a bolus driven, adaptive control method that uses prior data to update the patient specific parameters

\section{RESUlTS AND DisCUSSION}

\section{A. Trial A}

Patient A was a 76 year old male post coronary artery bypass surgical patient admitted into the ICU 33 days before the trial. The results are presented in Fig. 1. The patient was 


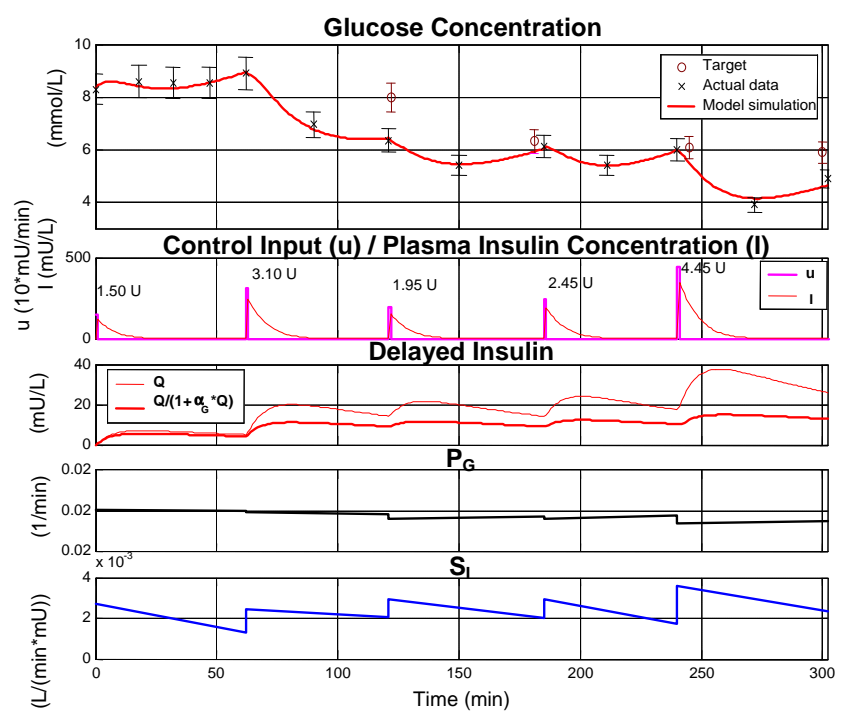

Fig. 1. Trial A progression

diagnosed with respiratory failure secondary to mediastinal sepsis and heart failure. The trial started with $G_{E}=8.3$ $\mathrm{mmol} / \mathrm{L}$ and the targeted hourly reduction was $10 \%$. This first trial used $\alpha_{G}=1 / 25 \mathrm{~L} / \mathrm{mU}$, effectively seeing the patient as having a lower glucose clearance saturation limit.

Insulin sensitivity, $S_{I}$, took on a saw tooth shape throughout this trial as a result of compensating for an incorrect insulin half-life parameter, $k$. The protocol for modifying $k$ was introduced after this trial. When the halflife was corrected, the fitted $S_{I}$ became smoother and more physiologically valid. At $240 \mathrm{~min}$, the controller demanded a larger bolus than the two previous injections (4.45 vs. 1.95 and $2.45 \mathrm{U})$ to achieve a similar percentage reduction because the model saw the patient as being at the saturation limit, thus requiring much more insulin to achieve the desired level. Although the patient did not suffer a hypoglycemic episode, the saturation limit was raised, with $\alpha_{G}$ lowered to $1 / 65 \mathrm{~L} / \mathrm{mU}$ for patient safety.

\section{B. Trial B}

Patient B was a 77-year-old female admitted to the ICU 30 days before the trial, diagnosed with diarrhea and sepsis due to lung injury. The trial began with $G_{E}=11.65 \mathrm{mmol} / \mathrm{L}$, and the targeted hourly reduction was $15 \%$. The results are presented in Fig. 2.

This trial demonstrated the impact of real-time modification of the insulin effective half-life, $k$. The data collected from the first two hours resulted in a saw tooth shape for $S_{I}$ similar to Trial A. The effective insulin half-life was automatically modified to 35 minutes at $120 \mathrm{~min}$ to eliminate the non-physiological variation of $S_{I}$ from a grossly incorrect assumed half-life. The immediate impact of this alteration is a much smoother and more

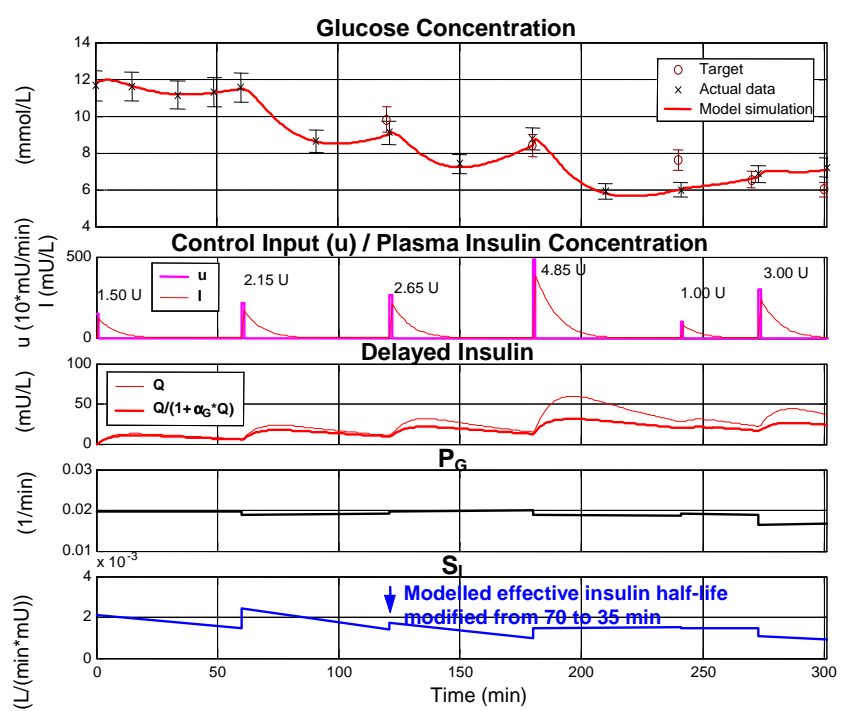

Fig. 2. Trial B progression

physiologically realistic $S_{I}$ variation from 120 min onwards, together with much lower resulting target errors.

The target reduction for 240 and 300 min were set lower than $15 \%$ because the controller predicted that glucose clearance saturation would limit the achievable drop. However, this patient probably had a higher saturation limit than assumed, or $\alpha_{G}$ higher than $1 / 65 \mathrm{~L} / \mathrm{mU}$. Hence, these two target errors are larger. An extended trial would have allowed the identification of a more correct value.

\section{Trial C}

Patient $\mathrm{C}$ was a 62-year-old male Type 2 diabetic admitted to the ICU 6 days before the trial with an acute abdominal aortic aneurysm. The trial started with $G_{E}=10.3$ $\mathrm{mmol} / \mathrm{L}$, and the targeted hourly reduction was $15 \%$. The results are shown in Fig. 3.

This patient's modeled $p_{G}$ and $S_{I}$ are both very smooth with minor discontinuity between each fit. This result indicates that the generic parameters used were similar to the actual patient values. The first two target glucose errors are larger because of the rapid decline in $S_{I}$ from 0 to 120 min due to the onset of the trial or the controller adapting from initial values. During this time, the patient's metabolism might have undergone alterations caused by the introduction of significant exogenous insulin, or the patient was becoming more agitated, triggering increased stress hormone secretion, and therefore reducing insulin sensitivity. The last two predictions had minimal error, showing that the controller had 'dialed in' and adapted to match the patient's dynamics. 


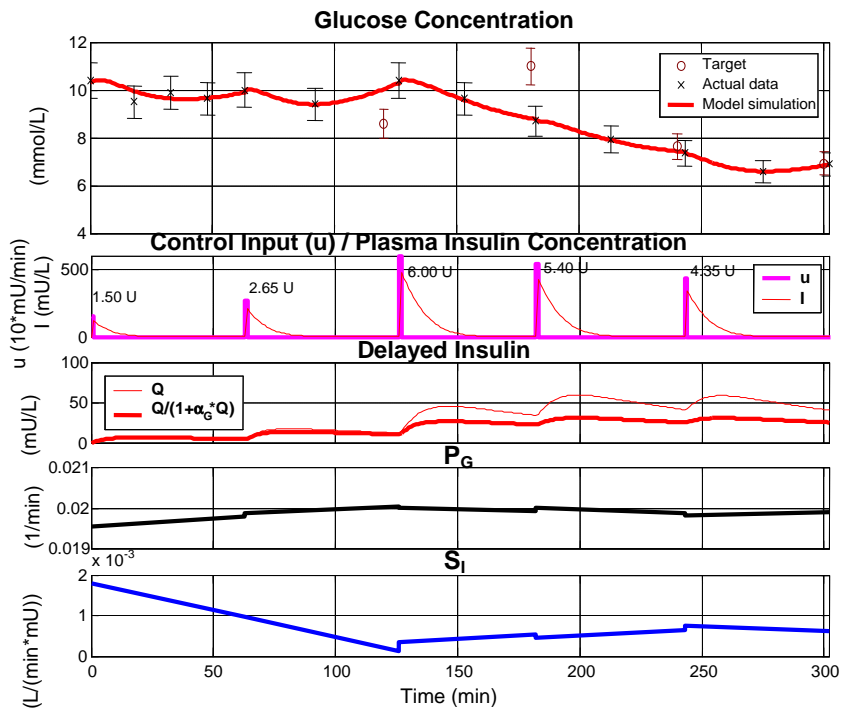

Fig. 3. Trial C progression

\section{CONCLUSIONS}

The clinical trials conducted during this study demonstrate the potential of the control algorithm designed for set-point regulation of hyperglycemia across a range of critically ill patients. The model and algorithm developed are capable of capturing a patient's glucose-insulin system dynamics, despite inter-patient variability and time varying physiological condition. The physiologically justified, nonlinear mathematical model developed accounts for nonlinear saturation of plasma insulin disappearance rate and its saturable utilization to reduce blood glucose levels. Further investigation into glucose clearance saturation limit and methods of real-time adaptation should permit the controller to obtain better performance. The addition of delayed insulin utilization via a convolution integral has accounted for the accumulation dynamic seen in prior clinical trials [10]. Removing endogenous insulin production from the model reduced model complexity and resulted in a unique metabolic glucose resistance index, $p_{G}$. Finally, an integration-based method is used for identifying patient specific time-varying parameters in real-time, resulting in a convex, computationally simple, linear, least squares solution. Overall, the research presented is a significant step towards more fully automated adaptive control of hyperglycemia in critically ill patients.

\section{REFERENCES}

[1] S. E. Capes, D. Hunt, K. Malmberg, and H. C. Gerstein, "Stress hyperglycemia and increased risk of death after myocardial infarction in patients with and without diabetes: a systematic overview," Lancet., Vol. 355, no. 9206, pp. 773-778, 2000.
[2] D. B. Coursin, and M. J. Murray, "How sweet is euglycemia in critically ill patients?" Mayo. Clin. Proc., vol. 78, no. 12, pp. 1460-1462, 2003.

[3] J. S. Krinsley, "Association between hyperglycemia and increased hospital mortality in a heterogeneous population of critically ill patients," Mayo. Clin. Proc., vol. 78, no. 12, pp. 1471-1478, 2003.

[4] K. C. McCowen, A. Malhotra, and B. R. Bistrian, "Stressinduced hyperglycemia," Crit. Care. Clin., vol. 17, no. 1, pp. 107-124, 2001.

[5] G. Van den Berghe et al., "Intensive insulin therapy in the critically ill patients," N. Engl. J. Med., vol. 345, no. 19, pp. 1359-1367, 2001.

[6] G. Van den Berghe et al., "Outcome benefit of intensive insulin therapy in the critically ill: insulin dose versus glycemic control," Crit. Care. Med., vol. 31, no. 2, pp. 359-366, 2003.

[7] J. S. Krinsley, "Decreased mortality of critically ill patients with the use of an intensive glycemic management protocol," presented at the SCCM 33rd Annual Congress, 2004.

[8] R. Hovorka et al., "Partitioning glucose distribution/transport, disposal, and endogenous production during IVGTT," Am. J. Physiol. Endocrino.l Metab., vol. 282, pp. E992-E1007, 2002.

[9] F. Chee, T. Fernando, and P. V. van Heerden, "Closed-loop control of blood glucose levels in critically ill patients," Anaesth. Intensive Care, vol. 30, no. 3, pp. 295-307, 2002.

[10] C. V. Doran, J. G. Chase, G. M. Shaw, K. T. Moorhead, and N. H. Hudson, "Automated insulin infusion trials in the ICU," Diabetes Technology and Therapeutics, vol. 6, no. 2, 2004.

[11] R. N. Bergman, D. T. Finegood, and M. Ader, "Assessment of insulin sensitivity in vivo," Endocr. Rev., vol. 6, no. 1, pp. 45-86, 1985.

[12] A. Natali et al., "Dose-response characteristics of insulin action on glucose metabolism: a non-steady-state approach," Am. J. Physiol. Endocrinol. Metab., vol. 278, no. 5, pp. E794-801, 2000.

[13] R. L. Prigeon, M. E. Roder, D. Porte Jr., and S. E. Kahn, "The effect of insulin dose on the measurement of insulin sensitivity by the minimal model technique. Evidence for saturable insulin transport in humans," J. Clin. Invest., vol. 97, no. 2, pp. 501-507, 1996.

[14] B. Thorsteinsson, "Kinetic models for insulin disappearance from plasma in man," Dan. Med. Bull., vol. 37, no. 2, pp. 143153, 1990.

[15] K. Ellemann et al., "Kinetics of insulin disappearance from plasma in cortisone-treated normal subjects," Clin. Endocrinol. (Oxf.), vol. 26, no. 5, pp. 623-628, 1987.

[16] A. Caumo et al., "Undermodeling affects minimal model indexes: insights from a two-compartment model," Am. J. Physiol., vol. 276, no. 6 pt. 1, pp. E1171-1193, 1999.

[17] F. Bettini, A. Caumo, and C. Cobelli, "Minimal models in meallike protocols: simulation studies to assess precision and physiological plausibility of parameter estimates," presented at the IEEE 17th Annu. Conference, 20-23 Sept. 1995.

[18] C. E. Hann, and M. S. Hickman Projective, "Curvature and Integral Invariants," Acta Applicandae Mathematicae, vol. 74, pp. 177-193, 2002.

[19] R. Hovorka, and P. Vicini, "Parameter estimation" In Modelling Methodology for Physiology and Medicine, E. Carson, and C. Cobelli, Eds. London, Academic Press, 2001, pp. 107-151.

[20] R. N. Bergman, R. Prager, A. Volund, and J. M. Olefsky, "Equivalence of the insulin sensitivity index in man derived by the minimal model method and the euglycemic glucose clamp," J. Clin. Invest., vol. 79, no. 3, pp. 790-800, 1987.

[21] C. Cobelli, A. Caumo, and M. Omenetto, "Minimal model SG overestimation and SI underestimation: improved accuracy by a Bayesian two-compartment model," Am. J. Physiol., vol. 277, no. 3 pt. 1, pp. E481-488, 1999.

[22] R. A. DeFronzo, J. D. Tobin, and R. Andres, "Glucose clamp technique: a method for quantifying insulin secretion and resistance," Am. J. Physio., vol. 237, no. 3, pp. E214-223, 1979.

[23] GlucocardTM Test Strip 2 Data Sheet, Arkray Inc., Japan, 2001. 